\title{
Development and Application of Artificial Intelligence
}

\author{
Yingjie Wang ${ }^{1-2, a}$ \\ ${ }^{1}$ College of Information Engineering, Dalian University, Dalian 116622, P . R. China \\ ${ }^{2}$ Software School, Dalian University of Technology, Dalian 116621, P . R. China \\ ayingjiewang2008@163.com
}

\begin{abstract}
Keywords: Artificial Intelligence, Robotics, Learning System
Abstract. Since the successes of the Artificial Intelligence might increasingly impact on multiple subjects in new millennium, it is necessary to write a summary of the recent development of this technology. This paper refined four essential and important aspects of the Artificial Intelligence, summarized both successful cases and the viewpoints of researchers, and evaluated the cases on different laterals. Development of autonomous agent in the application areas, such as self-driving car and robotics should improve their performance. Biological computer technology with intelligence might be playing a key role in Computer Science area in future.
\end{abstract}

\section{Introduction}

There still is an argument about whether the intelligence can be manufactured. In 1956, the Artificial Intelligence (AI) discipline was given the birth after about one-month symposium which had been taken part in by those experts and academics who were interested in machine intelligence. During more than half a century development, Artificial Intelligence has already applied in multiple scientific areas, which include recognition technology, automatic engineering, and knowledge engineering. In recent 12 years, the computational intelligence such as artificial life, immune computation and evolutionary computation, has been gradually developed and applied all over the world. The applications of the machine intelligence, such as robotics and driverless car, were being developed and exhibited with new performance. In addition, a learning system could obtain knowledge online and it can be working well for more than one year or longer if it is running on a stable computer. In short, Artificial Intelligence shows a variety of amazing experiences from a machine.

Applications of Artificial Intelligence technology into industry might result in many new developments of technology areas. This paper will summarize following Artificial Intelligence aspects in several technology areas: the autonomous agent with Google's driverless car as an example, the robotics such as ASIMO and BigDog, the supercomputer like Watson, and the machine intelligence (learning system) taking Never-Ending Language Learning System (NELL) as an example.

\section{Autonomous agent}

(1) Google's Driverless Car

The autopilot technology might be impacting on the development of vehicles as well as of aircrafts, since the progress of Artificial Intelligence on relatively complex traffic situation might become quite mature today. The driverless Cars, which could be much safer than human-driving vehicles, have been built by Google, because at least 1.2 million lives (from World Health Organization) could be saved from the traffic accidents if the Artificial Intelligence technology can help human operate cars. Those self-driving cars controlled by computers have been recorded no any traffic accident after they ran about 300,000 miles. However, those cars have been tested on Google's secret racecourse more than real-road conditions. Urmson [1], a Google engineer, believes that they still need to be improved to handle temporary signals or snow weather. In sum, Google exhibits a pretty outstanding and successful beginning to combine Artificial Intelligence (related to Autonomous Agent) with automobile technology. 
(2) Research of Sebastian Thrun

AI software plays a key role during autonomous driving process. Three main aspects of autonomous agent including perception, planning and control are managed by the software [2].The self-driving cars via sensors provide the necessary perception information to the self-driving system. In addition, the system might also require associating with local map, GPS (Global Positioning System) and INS (Inertial Navigation System). According to that information, the software of system will make a path planning. Then the path planning will direct the control system. The software, which designed with Artificial Intelligence cooperating with vehicle hardware, effects on over all self-driving period.

(3) Evaluation

The self-driving cars should be enhanced to install displacement sensors to serve the movement information for navigation system. In case, the GPS signal and INS are not available for long time, for example, in a long tunnel with branches, just like Liverpool to Birkenhead Tunnel, the system can pilot the car on a right way.

\section{Robotics}

(1) ASIMO

As Honda introduced, "ASIMO is the first humanoid robot in the world that can walk independently and climb stairs." ASIMO could be not only able to walk, as human does, but also can understand the gestures and voice commands which were set up in advance. Firstly, the moving of robots was a bottle-neck in the development of robotics. The ASIMO took a great step on the moving method of robot. It realised smooth turning by leaning the body to strike a balance and reduce the resistance force during its moving. What's more, ASIMO has capability of predicting turns so that it can conduct the body to act before the turn comes. Another innovation in robot moving goes for its high-level degree of freedom of which the components function as human joints and muscles to guarantee the flexibility of ASIMO's movement. Secondly, the sense system of ASIMO is also superior to that of its precedents, especially the vision system. Acting as eyes, the two basic video cameras in head of ASIMO "use stereoscopic vision and a proprietary vision algorithm" to capture images, recognize items, calculate distance and keep the ASIMO moving safely under an undesirable environment [3]. The ultrasonic sensors also contribute to this advanced vision system by finding objects and correcting the differences between the captured images of real environment and those in his database. As far as now, ASIMO is still not an autonomous robot, but an intelligent one. To be specific, it cannot think about the ideas on its own and then carry them out. The actions of ASIMO must depend on either human control or the pre-set programmes of a specific job within its understanding. As for the powering system, ASIMO lives upon a rechargeable, 51.8 volt lithium ion (Li-ION) battery. Put into the backpack that ASIMO carries, the battery weighs 13 pounds and could provide an hour energy at one time for the robot, which indicates that if ASIMOS is likely to do a long-lasting task then it is necessary for researchers to prepare extra batteries.

\section{(2) Big Gog}

Initiating a robot that can go wherever human and animal go, the Boston Dynamics set up a program funded by Tactical Technology Office at DARPA and created a BigDog, the alpha male robots [4]. This robot fits in a rough-terrain working environment, for instance, walking across rubble, running in snow and water, or hiking through a muddy, and use an environmental-friendly method such as hydraulic actuation system to supply his action. It could keep an excellent balance even someone tries to push it away impertinently. Multifunctional and bionic, the BigDog has four legs, as most vertebrate animals do, which allow it to walk, run, climb and carry heavy loads and a figure of which looks like a large dog, so named BigDog.

An efficient robot requires an efficient control system. In order to well navigate the dog and regulate its energy consumption under different circumstances, the central control system could take charge of movement and handle variety sensors, which would be responsible for sub-controls and serve necessary perceptions while the internal states of BigDog and the condition of energy supply 
system are for other sensors. An efficient control system help the BigDog get a great test results. BigDog can run at about 4 miles per hour.

(3) Summary of Researchers

It is still an argument whether it is necessary that we need to make a robot, which should be able to communicate with human rather than a senseless machine and apparently more like human. There is a known issue that robots might know nothing if they has not be programmed to know, and also they cannot do nothing if without program [5]. However, Hassler emphasizes that these robots could cross those field, where humans could not go, in dangerous to perform a task, instead of unreasonably exhibiting them like expensive curiosities or amusing toys. Spitznagel believe that the robots might become threat for employment if they can be as intelligent as workers, although they are not today.

(4) Evaluation

The ASIMO is not autonomous robot although it is definitely elegant and flexible on dancing or moving. In addition, there is no evidence shows whether the BigDog can autonomous or not. That means they are far away from human intelligence. It is obviously unnecessary to worry about if the artificial intelligence could exceed human intelligence. However, it should be the time to consider what should be the restriction of robotics, in case, we cannot dare to imagine if some objects with high- qualitative artificial intelligence will do something opposing human lives.

\section{Reasoning}

(1) Supercomputers - Watson

Based on the software construction of "Apache Hadoop which analyses the unstructured data to generate the index and the Apache UIMA which is a framework for arrangement of unstructured data", Watson demonstrated again the power of computer and of intelligent robot. IBM claims that the participation of DeepQA, a natural language processing program, could understand human sentence makes the Watson more compelling.

Researchers entered 200 million recording items and kept them in database for further researching for building an optimized knowledge database of Watson. The ever-faster memory bandwidth speed aids Watson in data processing so that the computer could search for matches and finally determine the best answer after analyse the relevant logic rules as soon as possible. In practice, given an instruction, the computer would act as a huge searching engine: analyzing the question, identifying the key words then looking for hints for the question answers. And that is the way by which Watson beat two human competitors in a famous quiz show Jeopardy. Although the computer is not able to use a voice-recognition technology during the show and the questions are required to be shown in text, Watson could speak out the answers in a synthesized voice.

(2) Summary of Researchers

The supercomputer could challenge human intelligence by using the Artificial intelligence technology. Russell and Norvig [6] state that in their book, "The representation of knowledge and the reasoning processes that bring knowledge to life are central to the entire field of artificial intelligence." The representation and reasoning system (RRS), which is composed of a language for communication, an approach to analyse the meaning of the language, and policies to retrieve answers, should be required, in order to enable reasoning and knowledge working with supercomputers. The RRS lets the computer (such as a supercomputer) understand something and associate the sentences with the some meaning in such language; therefore, the computer could answer the questions [7].

(3) Evaluation

Today, no computer with Artificial Intelligence could be able to match up with a human brain. Although the Watson could win the game by using huge memorizing capacity and calculating ability, it cannot do as well as human on other aspects. For example, it can only understand the text content instead of speech since it might not own the ability to recognize the oral language. However, it might become true that the intelligence of the computers(or supercomputer) could come up with human. Human has taken about 100,000 years to reach the current level of intelligence but the computer development has only spend several decades. The new generation computer (or computer cluster), 
which utilizes the biological technology and nerve network technology, has already been studying for a couple of years.

\section{Learning System}

\section{(1) NELL}

The computational learning system made a significant advance in 2010 , breaking through a long-existed development in semantics. Based on the database input by a research team sluggish of Carnegie Mellon University, a computer system "Never-Ending Language Learning System" (NELL) achieved in self-learning formal descriptions of concepts and relationships by scanning web pages and reached a relatively high accuracy of knowledge (87\%). Compared with former semantic learning system, the NELL is a more active learner: it can self-correct errors along with its research progress. However, it might still need researchers to label and correct its errors and find solutions to conquer its misconception of grammar modifiers and of terminology identification. At present, universities, government labs and some leading enterprises (including Google, Microsoft and IBM) also participate in the exploration of semantics, seeking to use it in future applications.

(2) Views of Researcher

Since the Artificial Intelligence concept was presented, as a part of this concept Machine Learning has gradually impacted on those explorations of multiple fields, such as, largely ignored computational considerations in statistics field, the web knowledge grasping via programming, and even, whether computers could learn to play games [8]. For dealing with the problems of learning processes, a discipline covering a large number and wide scope of subjects, which has produced fundamental statistical-computational theories, has designed learning algorithms. These algorithms could universally show the effect on a variety of tasks including the computer vision of business systems, the speech recognition for translating application and so on. Especially, the data mining in the Information Industry to perceive the regularities concealed behind an increasingly large amount of data on the web pages, like NELL, might have pivoted around the algorithms.

(3) Evaluation

Learning system is only a part of the machine learning. The whole picture of the machine learning includes much more related to mathematics and logic, such as, Density Estimation, Optimization, and Conditional Densities.

\section{Conclusion}

Artificial Intelligence, since 2000, does make a significant progress on several aspects but the researchers are still facing variety problems. By summarizing the successes with examples and illustrating case studies and some views of researchers and academics, this paper gives a relative explicit panorama of above aspects of Artificial Intelligence. This technology is increasingly impacting on other technology areas. Introduction of the essential points of Artificial Intelligence result in the theories to be comprehensible. Classifying the successes gives a schema of autonomous agent, robotics, supercomputer and machine learning aspects. Knowledge of those basic concepts shows that Artificial Intelligence technology heavily depends on software support. Examples related the successes reveal an increasing belt between Artificial Intelligence and other areas. Autonomous agents as a burgeoning direction offer human more and more new experiences. Robotics shows people a wide range of amazing behaviours although it has insufficiency. Supercomputer could answer almost any question since it won two former champions on Jeopardy game programme. In addition, a Learning System could get knowledge online restlessly.

Acknowledgment. This work is partially supported by the Liaoning Provincial Department of Education Science Research general project under Grant No. L2014496. 


\section{References}

[1] Urmson, C. The self-driving car logs more miles on new wheels. [online]. Available: http://googleblog.blogspot.co.uk/2012/08/the-self-driving-car-logs-more-miles-on.html\#!/2012 /08/the-self-driving-car-logs-more-miles-on.html [Accessed Sep 2015].

[2] Thrun, S. Toward Robotic Cars, ACM, April 2010. Vol.53 No.4

[3] Obringer, L. A. \& Strickland, J., How ASIMO Works, [online] Available: http://www.pcworld.com/article/219893/ibm_watson_vanquishes_human_jeopardy_foes.html [Accessed Sep 2015].

[4] Raibert, M., Blankespoor, K., Nelson, G., Playter, R. \& the BigDog Team BigDog, the Rough-Terrain Quaduped Robot, Boston Dynamics, Boston (2008).

[5] Alda, A. Robot Pals, Public Broadcasting Service, [online] Available: http://www.pcworld.com/article/219893/ibm_watson_vanquishes_human_jeopardy_foes.html [Accessed Oct 2015].

[6] Russell, S. J. \& Norvig, P. Artificial Intelligence: A Modern Approach (Second Edition), Pearson Education, New Jersey (2003).

[7] Poole, D., Mackworth, A. \& Goebel R. Computational Intelligence - A Logical Approach, Oxford University Press, New York (1998).

[8] Mitchell, T.M. The Discipline of Machine Learning, Carnegie Mellon University (2006). 